\title{
Development of chrysin loaded poloxamer micelles and toxicity evaluation in fish embryos
}

\author{
Tanongsak Sassa-deepaeng ${ }^{1}$, Surachai Pikulkaew ${ }^{1,2}$, Siriporn Okonogi ${ }^{1,3, *}$ \\ ${ }^{1}$ Nanoscience and Nanotechnology Program, the Graduate School, Chiang Mai University, Thailand; \\ ${ }^{2}$ Department of Food Animal Clinic, Faculty of Veterinary Medicine, Chiang Mai University, Chiang Mai, Thailand; \\ ${ }^{3}$ Department of Pharmaceutical Sciences, Faculty of Pharmacy, Chiang Mai University, Chiang Mai, Thailand.
}

\begin{abstract}
Summary Poloxamer micelles promise safety and efficacy for many water insoluble drugs. Chrysin has been reported to have anticancer, anti-inflammatory, antioxidant, and anti-aromatase activities but its water insoluble properties limit its pharmaceutical application. In the present study, chrysin loaded poloxamer micelles were developed. Two types of poloxamers, Pluronic F-68 and Pluronic F-127 were compared. It was found that chrysin loaded Pluronic F-68 micelles (CS-P68) and chrysin loaded Pluronic F-127 micelles (CS-P127) obviously increase the aqueous solubility of chrysin. The results also indicated that the type of polymer and ratio of drug to polymer affected size and desirable characteristics of the micelles. The micelle system of CS-P68 and CS-P127 formed at drug to polymer ratios of 1:4 and 1:2, respectively, was found to be the most suitable monodispersed system with a nanosize-range diameter. The in vivo study in zebrafish eggs indicates that the toxicity of CS-P68 and CS-P127 is a dose response. CS-P68 and CS-P127 at a drug dose of $10 \mathrm{ng} / \mathrm{mL}$ or less is safe for zebrafish embryo growth. The results of this study indicate enhanced water solubility of chrysin. Chrysin loaded poloxamer micelles are promising for further use in in vivo studies in mammalian animals and humans.
\end{abstract}

Keywords: Chrysin, poloxamer, pluronic, polymeric micelles, solubility

\section{Introduction}

Chrysin, a natural flavonoid compound with the IUPAC name 5,7-dihydroxy-2- phenyl-4H-chromen-4one, can be extracted from plants, honey, and propolis (1). It has been shown that chrysin is abundant in the fruit of Oroxylum indicum (2). The fruit of this plant is commonly used in Thailand and other East Asian countries as food and herbal medicine. Chrysin possesses several biological activities including, anti-cancer $(3,4)$, anti-inflammation $(5,6)$, and antioxidant properties $(7,8)$ and is reported to cause an increase of testosterone production via suppression of aromatase, an enzyme that converts androgen to estrogen (9). However, biological

Released online in J-STAGE as advance publication June 29, 2016.

*Address correspondence to:

Dr. Siriporn Okonogi, Department of Pharmaceutical Sciences, Faculty of Pharmacy, Chiang Mai University, Chiang Mai 50200, Thailand.

E-mail: siriporn.okonogi@cmu.ac.th actions are only facilitated given the stable structure and low molecular weights of the active compounds that can be soluble and pass through cell membranes (10). Unfortunately, chrysin has a major problem of solubility. Its insoluble aqueous property causes low absorption and low bioavailability. Therefore, it is essential to improve the solubility of chrysin in order to increase its pharmaceutical and medical applications.

Various techniques can be applied for enhancement of the solubility and dissolution rate of poorly water soluble drugs such as solubilization by cosolvents (11), salt formation (12), inclusion complex in cyclodextrins (13), solid dispersions (14-16), and micellization $(17,18)$. For chrysin, enhancement of its water solubility by cosolvents has been reported (19). However, the reported cosolvents used were dimethylformamide and tetrahydrofuran which are harmful organic solvents. Therefore, a search for better techniques particularly with nontoxic carriers is still challenge.

Among several solubilizing techniques, micellization using polymeric micelles seems to be most promising for solubility enhancement of drugs, because it 
overcomes the limitations of the other techniques $(20,21)$. Poloxamer is one of the most common polymers used to fabricate polymeric micelles $(22,23)$. Poloxamer is an amphiphilic block copolymer. The non-covalent incorporation of many drugs into the hydrophobic core of poloxamer micelles resulted in an increase of solubility, stability, and bioavailability of the drugs. Therefore, this biocompatible polymer has been used for the encapsulation of various water insoluble drugs into nanoparticles in the form of polymeric micelles (24). Moreover, poloxamer is reported to be biocompatible, with low toxicity, and low degradation $(25,26)$. In addition, poloxamer can minimize adsorption to surfaces due to hydrophilicity (27).

The aim of the present study is to develop chrysin loaded poloxamer micelles in order to enhance the water solubility of chrysin. The effect of polymer and solvent on the characteristics of the polymeric micelles obtained was investigated. Moreover, in vivo toxicity of the selected system of chrysin loading micelles on fish embryos was evaluated.

\section{Materials and Methods}

\subsection{Materials}

Chrysin was purchased from Sigma-Aldrich Co. (St. Louis, USA). Poloxamers (Pluronic F-68 and Pluronic F-127) were purchased from O-BASF Co. (Ludwigshafen, Germany). Tween 80 was obtained from Namsian Co. Ltd. (Bangkok, Thailand). Acetone was from RCI Labscan (Bangkok, Thailand). Ethanol was from Scharlau (Barcelona, Spain). All solvents were of analytical grade.

\subsection{Polymeric micelle preparation}

Two types of poloxamers; Pluronic F-68 and Pluronic F-127 were used in this study. Chrysin loaded polymeric micelles were prepared by dissolving chrysin separately in two different organic solvents; acetone or ethanol. The drug solution was added dropwise with Pluronic F-68 or Pluronic F-127 solution to obtain the mixture of chrysin-polymer at the weight ratios of $1: 1$, $1: 2,1: 3,1: 4,1: 5,1: 10$, and 1:15. After that, Tween 80 was added. Deionized water was added to volume and the mixture was frozen at $-20^{\circ} \mathrm{C}$ and subsequently lyophilized under vacuum for $24 \mathrm{~h}$. After lyophilization, the obtained dry chrysin loaded Pluronic F-68 and Pluronic F-127 micelles namely CS-P68 and CS-P127, respectively, were re-suspended in deionized water to the desired drug concentration for further studies.

\subsection{Determination of size, size distribution, and zeta potential of the micelles}

Size and size distribution (PDI) of CS-P68 and CS-
P127 was measured using Zetasizer NanoZS (Malvern Instruments, UK) working on the principle of photon correlation spectroscopy (PCS). A portion of $1 \mathrm{~mL}$ of the micelle dispersion in water was transferred into a quartz cuvette and exposed to laser light diffraction at an angle of $173^{\circ}$. The intensity of the peak that showed the highest population of the micelles of that size was recorded. Zeta potential of the micelles was measured using the same instrument and determined five times for each sample. Results were automatically calculated by the analyzer.

\subsection{In vivo toxicity study}

The in vivo toxicity experiment was done using a method described previously (28) with some modification. Briefly, 4-h of age fresh zebrafish eggs were gently filled into a series of $10 \mathrm{~mL}$ aqueous clear mixtures containing CS-P68 or CS-P127 with chrysin concentrations of $1,10,100,1,000$ and 10,000 ng/mL. The incubation temperature was $28^{\circ} \mathrm{C}$. The number of zebrafish eggs was 15 for each system. Water without any polymeric micelles was used as a control. The mortality of the embryos in each system was observed every $24 \mathrm{~h}$ for a period of $72 \mathrm{~h}$ under a stereo microscope (Nikon, Tokyo, Japan).

\subsection{Statistical analysis}

The preparation, size measurement, and toxicity study were done in triplicate and the results are expressed as mean \pm S.D. Statistical analysis was done by using ANOVA and $P$-value at a level of $95 \%$ confidence limit.

\section{Results and Discussion}

\subsection{Preparation of chrysin loaded micelles}

In the process of chrysin loaded micelles, Tween 80 has been added for incorporation into the micelles. Tween 80 is a hydrophilic non-ionic surfactant widely used in emulsification and solubilizing of substances in medicinal, pharmaceutical, and food products. Moreover, it is used in conjunction with nanoparticles to improve specific delivery (29). Tween 80 is reported to be adsorbed on the surface by interacting with specific receptors on the blood brain barrier luminal face, and then transported into the brain (30). Therefore, incorporation of Tween 80 into the polymeric micelles of poloxamer in the present study was to obtain the most desirable carrier for the chrysin solubilization and delivery system. It was found that chrysin could be loaded into both Pluronic F-68 and Pluronic F-127. The systems obtained after preparation were transparent aqueous dispersions. After lyophilization, the products obtained were still transparent but the state of matter was changed to a semisolid form as a gel- 
like product. After diluting with water, the semisolid products changed to transparent aqueous systems without any precipitation of chrysin. It was considered that all chrysin could be dissolved in the water. The result was in agreement with the previous results that the practically insoluble curcumin and xanthone could be solubilized by polymeric micelles and transparent aqueous mixtures obtained $(20,31)$.

\subsection{Effects of polymer and solvent types on size and size distribution of the micelles}

Two types of solvents, ethanol and acetone, were compared in the preparation of the micelles of two types of poloxamer. It was found that using ethanol as a solvent for chrysin in the preparation of drug loaded micelles yielded micelles with different sizes depending on the polymer type and drug to polymer ratio. The size and PDI as well as \% intensity of CS-P68 and CSP127 are shown in Table 1 and Table 2, respectively. It was found that the size of drug entrapped micelles was slightly larger than that of empty micelles for both polymers. The size of CS-P68 was in the range of 12.6-17.8 nm whereas that of CS-P127 was in the range of 11.2-14.1 $\mathrm{nm}$. The PDI was in the range of 0.1-0.2 for CS-P68 and 0.1-0.3 for CS-P127 indicating a good size distribution for both polymers. According to the peak intensity, the mixture at a weight ratio of 1:4 was considered to be the best formulation for CSP68 whereas that of 1:2 was considered to be the best formulation for CS-P127 because it showed a peak intensity of $100 \%$ as shown in Figure 1.

Using acetone instead of ethanol as a solvent for

Table 1. Characteristics of CS-P68 obtained from the use of ethanol as a solvent

\begin{tabular}{lccc}
\hline Ratio of chrysin to polymer & Size $(\mathrm{nm})$ & PDI & Intensity (\%) \\
\hline $0: 1$ & $10.4 \pm 3.4$ & 0.132 & 100.0 \\
$1: 1$ & $17.8 \pm 8.3$ & 0.204 & 98.5 \\
$1: 2$ & $12.6 \pm 4.2$ & 0.156 & 98.6 \\
$1: 3$ & $12.5 \pm 4.1$ & 0.147 & 98.6 \\
$1: 4$ & $12.7 \pm 4.1$ & 0.139 & 100.0 \\
$1: 5$ & $13.5 \pm 4.7$ & 0.192 & 96.3 \\
$1: 10$ & $14.3 \pm 5.6$ & 0.197 & 97.0 \\
$1: 15$ & $15.1 \pm 5.2$ & 0.263 & 95.3 \\
\hline
\end{tabular}

Table 2. Characteristics of CS-P127 obtained from the use of ethanol as a solvent

\begin{tabular}{lccc}
\hline Ratio of chrysin to polymer & Size (nm) & PDI & Intensity (\%) \\
\hline $0: 1$ & $9.1 \pm 2.2$ & 0.166 & 100.0 \\
$1: 1$ & $13.8 \pm 5.2$ & 0.179 & 96.8 \\
$1: 2$ & $11.7 \pm 3.2$ & 0.054 & 100.0 \\
$1: 3$ & $11.2 \pm 2.7$ & 0.195 & 97.6 \\
$1: 4$ & $13.3 \pm 3.9$ & 0.278 & 92.1 \\
$1: 5$ & $13.7 \pm 2.9$ & 0.247 & 79.7 \\
$1: 10$ & $14.1 \pm 3.6$ & 0.315 & 86.2 \\
$1: 15$ & $\mathrm{ND}$ & $\mathrm{ND}$ & $\mathrm{ND}$ \\
\hline
\end{tabular}

$\mathrm{ND}=$ not detectable. chrysin to formulate chrysin loaded polymeric micelles of both polymers CP-68 and CP-127 could also be obtained. Similarly to those using ethanol as a solvent, it was found that the size of chrysin loaded micelles was slightly larger than that of empty micelles. As shown in Table 3 and Table 4 for CS-P68 and CS-P127, respectively, it was found that the size of CS-P68 and CS-P127 was in the range of 10.5-16.8 $\mathrm{nm}$ and 10.1$14.5 \mathrm{~nm}$, respectively. It was observed that the size of chrysin loaded micelles prepared using acetone as a solvent for preparation of drug solution was slightly smaller but not significantly different than those using ethanol as a solvent. The PDI of the micelles of both polymers was in the same range as those prepared by using ethanol as a solvent. According to peak intensity,
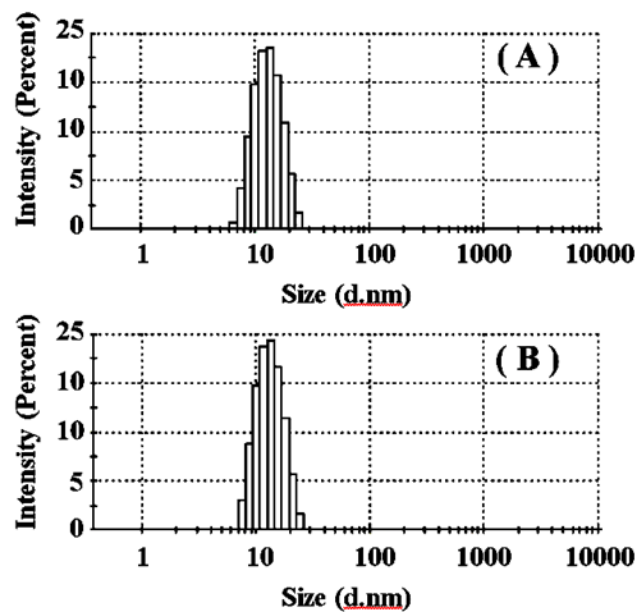

Figure 1. PCS analysis of CS-P68 (A) and CS-P127 (B) at drug to polymer ratios of $1: 4$ and $1: 2$, respectively obtained from the use of ethanol as a solvent.

Table 3. Characteristics of CS-P68 obtained from the use of acetone as a solvent

\begin{tabular}{lccc}
\hline Ratio of chrysin to polymer & Size (nm) & PDI & Intensity (\%) \\
\hline $0: 1$ & $10.2 \pm 2.9$ & 0.076 & 100.0 \\
$1: 1$ & $11.4 \pm 3.5$ & 0.112 & 97.9 \\
$1: 2$ & $10.7 \pm 3.6$ & 0.181 & 97.6 \\
$1: 3$ & $10.5 \pm 4.3$ & 0.126 & 100.0 \\
$1: 4$ & $12.1 \pm 4.3$ & 0.259 & 93.2 \\
$1: 5$ & $10.8 \pm 3.3$ & 0.215 & 96.8 \\
$1: 10$ & $12.4 \pm 5.2$ & 0.193 & 97.3 \\
$1: 15$ & $16.8 \pm 6.7$ & 0.217 & 96.3 \\
\hline
\end{tabular}

Table 4. Characteristics of CS-P127 obtained from the use of acetone as a solvent

\begin{tabular}{lrrr}
\hline Ratio of chrysin to polymer & Size (nm) & PDI & Intensity (\%) \\
\hline $0: 1$ & $9.1 \pm 2.3$ & 0.081 & 100.0 \\
$1: 1$ & $10.9 \pm 3.7$ & 0.138 & 98.8 \\
$1: 2$ & $11.8 \pm 3.9$ & 0.288 & 88.6 \\
$1: 3$ & $10.6 \pm 3.4$ & 0.099 & 100.0 \\
$1: 4$ & $10.1 \pm 2.5$ & 0.331 & 95.7 \\
$1: 5$ & $14.5 \pm 7.5$ & 0.193 & 98.7 \\
$1: 10$ & $11.8 \pm 4.3$ & 0.145 & 100.0 \\
$1: 15$ & $11.1 \pm 3.1$ & 0.066 & 100.0 \\
\hline
\end{tabular}



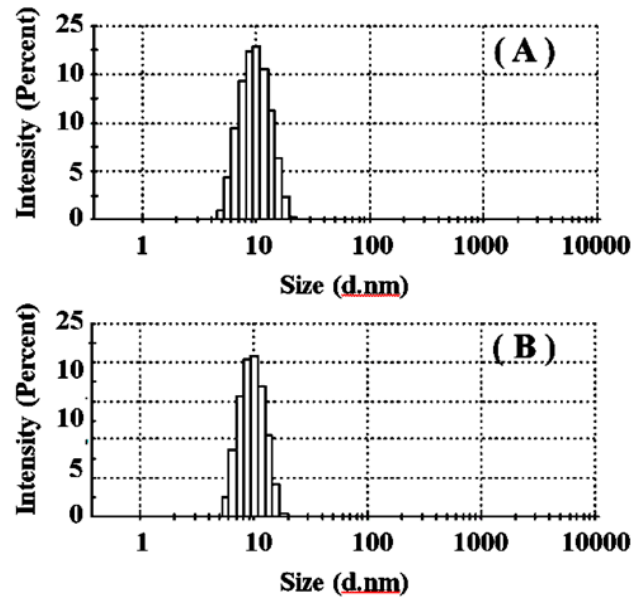

Figure 2. PCS analysis of CS-P68 (A) and CS-P127 (B) at drug to polymer ratio of $1: 3$ obtained from the use of acetone as a solvent.

the mixture at a weight ratio of 1:3 was considered to be the best formulation for CS-P68 because this system showed a peak intensity of $100 \%$ and showed a single size distribution peak as shown in Figure 2A. However, three systems of CS-P127 with drug to polymer ratios of $1: 3,1: 10$, and $1: 15$ showed a peak intensity of $100 \%$. Considering the particle size of these systems, it was found that the micelles at a ratio of 1:3 showed the smallest size of $10.6 \pm 3.4 \mathrm{~nm}$. The single size distribution peak of this system was obtained as shown in Figure 2B.

These results indicate that chrysin can be successfully entrapped in micelles of both types of poloxamers, Pluronic F-68 and Pluronic F-127. The size of CSP68 and CS-P127 obtained from all studied conditions are in the nanosize range. The results demonstrate that the types of polymers and the ratio of drug to polymer play an important role in the size of the developed drug loaded micelles whereas no significant difference between ethanol and acetone used as a solvent for drug dissolution was seen in the preparation process. It was found that the zeta potential of CS-P68 and CS-P127 from all conditions was approximately -12 to $-14 \mathrm{mV}$ (data not shown), indicating that the developed chrysin loaded polymeric micelles might have a possibility for aggregation. When comparing the developed chrysin loaded polymeric micelles to the intact chrysin added in water, it was found that clear aqueous systems of CS-P68 and CS-P127 were obtained whereas the intact chrysin at the same concentration precipitated in water. This result obviously indicates that water solubility of chrysin was increased dramatically when formed as CS-P68 and CS-P127. As poloxamer is composed of hydrophilic polyethylene oxide (PEO) and lipophilic polypropylene oxide (PPO) blocks, arranged in a PEOmPPOnPEOm structure (32), it can self-assemble into micelles in aqueous solution forming the hydrophobic PPO core surrounded by the hydrophilic PEO. The increased water solubility of chrysin using these polymeric micelles is

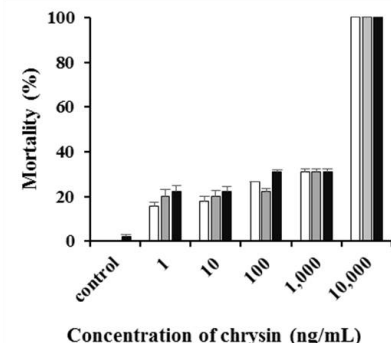

Figure 3. Mortality of zebrafish eggs at $24 \mathrm{~h}$ (white column), $48 \mathrm{~h}$ (gray column), and $72 \mathrm{~h}$ (black column) exposure to CS-P68.

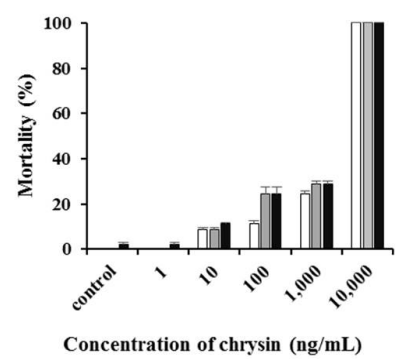

Figure 4. Mortality of zebrafish eggs at $24 \mathrm{~h}$ (white column), $48 \mathrm{~h}$ (gray column), and $72 \mathrm{~h}$ (black column) exposure to CS-P127.

considered to be due to the incorporation of chrysin into the hydrophobic portion of the micelles.

\subsection{In vivo toxicity of chrysin loaded micelles}

Regarding the solvent used, both ethanol and acetone could yield chrysin loaded micelles with a similar nano-size range but ethanol is considered to be a better solvent than acetone from the view point of environmental and human safety. Therefore, in the investigation of in vivo toxicity, only CS-P68 and CS-P127 with the proper ratio of drug to polymer of $1: 4$ and $1: 2$, respectively, and prepared using ethanol as a solvent were used. Chrysin has been reported to suppress an enzyme that converts androgen to estrogen resulting in an increase of testosterone (9). Therefore, it might be useful to know the safe dose or the maximum concentration of CS-P68 and CS-P127 which is considered as safe. In the present study, the embryo of zebra fish was used as a model for testing toxicity of the developed CS-P68 and CS-P127. The toxicity results expressed as mortality of zebrafish embryo are shown in Figure 3 for CS-P68 and Figure 4 for CS-P127. From these figures, it was noted that the mortality of the embryos with CS-P68 was higher than that with CS-P127 indicating that CS-P68 had higher toxicity than CS-P127. This effect was obviously seen particularly at the low dose range of $1-100 \mathrm{ng} / \mathrm{mL}$. However, toxicity of both micelles was not significantly different at a concentration of $1000 \mathrm{ng} / \mathrm{mL}$ or more. It was found that the toxicity of all samples was seen in a dose dependent manner. A $10 \mathrm{ng} / \mathrm{mL}$ dose or less was 


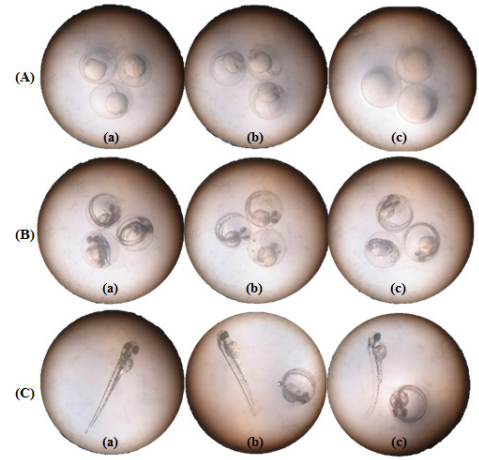

Figure 5. Morphology of surviving zebrafish embryos at 24 $\mathrm{h}(\mathrm{A}), 48 \mathrm{~h}(\mathrm{~B})$, and $72 \mathrm{~h}$ (C) exposure to water (a), CS-P68 at drug concentration of $1,000 \mathrm{ng} / \mathrm{mL}(\mathrm{b})$, and CP-127 at drug concentration of $1,000 \mathrm{ng} / \mathrm{mL}$ (c).

found to be safe for zebrafish embryos as less than $10 \%$ mortality was observed whereas doses of $100-1,000$ $\mathrm{ng} / \mathrm{mL}$ could be classified as a mild toxic dose as $10 \%$ to less than $30 \%$ mortality was observed. Higher than $1,000 \mathrm{ng} / \mathrm{mL}$ could be classified as moderate to severe toxicity to zebrafish embryos as $30-100 \%$ mortality was observed. The results also showed that the toxic effect of CS-P68 and CS-P127 was not time dependent. After incubating zebrafish eggs in the fixed drug dose systems but at different incubation times of 24,48 , and $72 \mathrm{~h}$, the mortality of the fish was not significantly different at each dose. The results revealed that no zebrafish embryos were found after $24 \mathrm{~h}$ exposure to $10,000 \mathrm{ng} / \mathrm{mL}$ whereas more than $70 \%$ of the embryos developed normally (same as control) after exposure to $1-100 \mathrm{ng} / \mathrm{mL}$. However, at a concentration of 1,000 ng/ $\mathrm{mL}$, some embryos (about $27 \pm 1 \%$ ) were dead after 24 $\mathrm{h}$ of exposure, the remaining embryos could develop but some showed delayed development. This effect could be seen clearly as shown in Figure 5. After 72 h, all embryos in the control group could hatch normally whereas only approx. $55 \pm 5 \%$ of the eggs exposed to CS-P68 and CS-P127 (1,000 ng/mL) showed normal development like in the control group but the remaining embryos were still at an early stage. They were not dead but showed slow development as seen in Figure 5C. It was considered that polymer might be associated with the toxicity of CS-P68 and CS-P127. In CS-P68, the drug to polymer ratio was $1: 4$ whereas in CS-P127 it was 1:2. The amount of polymer in CS-P68 was 2 times higher than that in CS-P127. Therefore, high mortality particularly at low drug concentrations caused by CSP68 was considered to be due to high concentration of polymer used in CS-P68.

\section{Conclusion}

Chrysin can be loaded in the polymeric micelles of Pluronic F-68 and Pluronic F-127. The obtained chrysin loaded micelles can increase its water solubility dramatically. The type of polymer and the ratio of drug to polymer play an important effect on size and desirable characteristics of the obtained micelles. The suitable chrysin loaded polymer micelles is composed of 1:4 and 1:2 of drug to Pluronic F-68 and to Pluronic F-127 ratios, respectively. The toxicity of these micelles is dose dependent but not time dependent. Chrysin at concentrations that do not exceed $10 \mathrm{ng} / \mathrm{mL}$ is considered safe for zebrafish embryos. The micelles with higher polymer ratios cause higher toxicity to the fish.

\section{Acknowledgements}

This study was financially supported by Rajamangala University of Technology Lanna (RMUTL). The authors would like to thank the Graduate School of Chiang Mai University for partial support. We also thank Faculty of Veterinary Medicine and Faculty of Pharmacy, Chiang Mai University for facility and instrument supports.

\section{References}

1. Samarghandian S, Afshari JT, Davoodi S. Chrysin reduces proliferation and induces apoptosis in the human prostate cancer cell line pc-3. Clinics. 2011; 66:1073-1079.

2. Raghu AV, Satheesh George S, Renju KV, Sindhu KK. Bioactive properties of phenolics present in Oroxylum indicum - A review. J Pharmacog Phytochem. 2013; 2:2327.

3. Sun LP, Chen AL, Hung HC, Chien YH, Huang JS, Huang CY, Chen YW, Chen CN. Chrysin: A histone deacetylase 8 inhibitor with anticancer activity and a suitable candidate for the standardization of Chinese propolis. J Agric Food Chem. 2012; 60:11748-11758.

4. Xuan HZ, Zhang JH, Wang YH, Fu CL, Zhang W. Antitumor activity evaluation of novel chrysin-organotin compound in MCF-7 cells. Bioorg Med Chem Lett. 2016; 26:570-574.

5. Ahad A, Ganai AA, Mujeeb M, Siddiqui WA. Chrysin, an anti-inflammatory molecule, abrogates renal dysfunction in type 2 diabetic rats. Toxicol Appl Pharm. 2014; 279:1-7.

6. Yao J, Jiang M, Zhang Y, Liu X, Du Q, Feng G. Chrysin alleviates allergic inflammation and airway remodeling in a murine model of chronic asthma. Int Immunopharmacol. 2016; 32:24-31.

7. Anitha TA, Rajadurai M. Antioxidative potential of chrysin, a flavone in streptozotocin-nicotinamide-induced diabetic rats. Biomed Prev Nutr. 2014; 4:511-517.

8. Patel RV, Mistry B, Syed R, Rathi AK, Lee YJ, Sung JS, Shinf HS, Keum YS. Chrysin-piperazine conjugates as antioxidant and anticancer agents. Eur J Pharm Sci. 2016; 88:66-177.

9. Jana K, Yin X, Schiffer RB, Chen JJ, Pandey AK, Stocco DM, Grammas P, Wang X. Chrysin, a natural flavonoid enhances steroidogenesis and steroidogenic acute regulatory protein gene expression in mouse Leydig cells. J Endocrinol. 2008; 197:315-323.

10. Ososki AL, Kennelly EL. Phytoestrogens: A review of the present state of research. Phytother Res. 2003; 17:845869.

11. Jeffrey WM, Alvarez-Nunez A, Yalkowsky SH. 
Solubilization by cosolvents. Establishing useful constants for the log/linear model. Int J Pharm. 2002; 245:153-166.

12. Serajuddin AT. Salt formation to improve drug solubility. Adv Drug Deliv Rev. 2007; 59:603-616.

13. Ali SM, Upadhyay SK, Maheshwari A. NMR spectroscopic study of the inclusion complex of desloratadine with betacyclodextrin in solution. Spectroscopy 2007; 21:177-182.

14. Okonogi S, Puttipipatkhachorn S. Dissolution improvement of high drug loaded solid dispersion. AAPS PharmSciTech. 2006; 7:E1-E6.

15. Oguchi T, Tozuka Y, Okonogi S, Yonemochi E, Yamamoto K. Improved dissolution of naproxen from solid dispersions with porous additives. Yakuzaigaku 1997; 57:168-173.

16. Chen Y, Zhang GGZ, Neilly J, Marsh K, Mawhinney D, Sanzgiri YD. Enhancing the bioavailability of ABT963 using solid dispersion containing Pluronic F-68. Int J Pharm. 2004; 286:69-80.

17. Rao VR, Nerurkar M, Pinnamaneni S, Rinaldi F, Raghavan K. Co-solubilization of poorly soluble drugs by micellization and complexation. Int J Pharm. 2006; 319:98-106.

18. Parekh P, Singh K, Marangoni DG, Bahadur P. Micellization and solubilization of a model hydrophobic drug nimesulide in aqueous salt solutions of Tetronic T904. Colloid Surf. B. 2011; 83:69-77.

19. Feizi S, Jabbari M, Farajtabar A. A systematic study on solubility and solvation of bioactive compound chrysin in some water + cosolvent mixtures. J Mol Liq. 2016; 220:478-483.

20. Naksuriya O, Shi Y, Van Nostrum CF, Anuchapreeda S, Hennink WE, Okonogi S. HPMA-based polymeric micelles for curcumin solubilization and inhibition of cancer cell growth. Eur J Pharm Biopharm. 2015; 94:501512.

21. Biswas S, Kumari P, Lakhani PM, Ghosh B. Recent advances in polymeric micelles for anti-cancer drug delivery. Eur J Pharm Sci. 2016; 83:184-202.

22. Al-Saden AA, Whateley TL, Florence AT. Poloxamer association in aqueous solution. J Colloid Interf Sci. 1982; 90:303-309.

23. Oh KT, Bronich TK, Kabanov AV. Micellar formulations for drug delivery based on mixtures of hydrophobic and hydrophilic Pluronic block copolymers. J Control Release 2004; 94:411-422.

24. Sahu A, Kasoju N, Goswami P, Bora U. Encapsulation of curcumin in Pluronic block copolymer micelles for drug delivery applications. J Biomater Appl. 2011; 25:619639.

25. Mayol L, Quaglia F, Borzacchiello A, Ambrosio L, Rotonda MIL. A novel poloxamers/hyaluronic acid in situ forming hydrogel for drug delivery: Rheological, mucoadhesive and in vitro release properties. Eur J Pharm Biopharm. 2008; 70:199-206.

26. Morlan ZU, Rios RC, Montes AC, Contreras LMM, Segundo EP, Quintanar AG, Guerrero DQ. Determination of poloxamer 188 and poloxamer 407 using highperformance thin-layer chromatography in pharmaceutical formulations. J Pharm Biomed Anal. 2008; 46:799-803.

27. Jindal N, Mehta SK. Nevirapine loaded Poloxamer 407/Pluronic P123 mixed micelles: Optimization of formulation and in vitro evaluation. Colloid Surf. B. 2015; 129:100-106.

28. Pamanji R, Yashwanth B, Bethu MS, Leelavathi S, Ravinder K, Venkateswara J. Toxicity effects of profenofos on embryonic and larval development of zebrafish (Danio rerio). Environ Toxicol Pharmacol. 2015; 39:887-897.

29. Sun W, Xie C, Wang H, Hu Y. Specific role of polysorbate 80 coating on the target of nanoparticles to the brain. Biomaterials. 2004; 25:3065-3071.

30. Prabhakar K, Afzal SM, Surender G, Kishan V. Tween 80 containing lipid nanoemulsions for delivery of indinavir to brain. Acta Pharm Sin B. 2013; 3:345-353.

31. Khonkarn R, Mankhetkorn S, Talelli M, Hennink WE, Okonogi S. Cytostatic effect of xanthone-loaded mPEG$b$-p(HPMAm-Lac ${ }_{2}$ ) micelles towards doxorubicin sensitive and resistant cancer cells. Colloid Surface B. 2012; 94:266-273.

32. Chiappetta DA, Sosnik A. Poly(ethylene oxide)poly(propylene oxide) block copolymer micelles as drug delivery agents: Improved hydrosolubility, stability and bioavailability of drugs. Eur J Pharm Biopharm. 2007; 66:303-317.

(Received June 3, 2016; Revised June 12, 2016; Accepted June 18, 2016) 